\title{
DE-NOISING OF TOMATO FRUIT IMAGE USING SPIRAL SEED FILTER
}

\author{
S. Kavitha \\ Research Scholar \\ Department of Computer Science \\ L.R.G. Government Arts College for Women \\ Tirupur \\ kavi.sportive@gmail.com
}

\author{
Dr. K. Sarojini \\ Assistant Professor \& Head of the Department \\ Department of Computer Science \\ L.R.G. Government Arts College for Women \\ Tirupur \\ saromaran@gmail.com
}

\begin{abstract}
:
Fruit disease causes more economic losses in agricultural industry. In prediction of disease image pre-processing plays an important role. Fruits may appear healthy and fresh to human eye but its quality is known by customer after eating the fruits. Images are used to forecast quality of the fruits and vegetables, but accuracy of grading will be affected by distortion. Various noise affect the quality of the image and it can be denoised by various filters. The preservative edges, background information and contrast of images are the challenging issues in exiting filtering methods. This research proposed Spiral Seed Filter (SSF) to increase the quality of the tomato fruit image by extracting the luma variance and by applying the row wise and column wise $3 \times 3$ cross correlation. The result shows that the proposed filter increases the PSNR (Peak Signal to Noise ratio) and reduces MSE (Mean Square Error) metric values and yield good results. It gives highest PSNR value such as 94.68. It gives 0.0001 as MSE value for proposed method.
\end{abstract}

Keywords: Image processing, Filter, PSNR (Peak Signal to Noise ratio), MSE (Mean Square Error)

\section{Introduction}

Tomato belongs to the family of Solanum lycopersicum L. Tomato is the second largest fruit consumed worldwide. Tomato is consumed throughout the world for its nutrition contents. Thus cultivation of tomato has increased in recent years. Tomato fruit is of four types as milano, chonto, cherry and industrial. Among these Milano has the high commercial value as the shelf life is comparatively good [1]. Chonto and cherry are consumed fresh and mostly consumed in domestic. Tomato rich in vitamin $\mathrm{C}$ and $\mathrm{A}$, Calcium, Phosphorus, Potassium, Iron and Copper which supports in human metabolism. Tomato is the most widely cultivated crop in India. Winter and summer seasons are the most suitable seasons for cultivation of tomato crop as it cannot withstand frost. Average temperature ranging from $21^{\circ}$ $23^{\circ} \mathrm{C}$ is the most suitable weather for good yield of fruit. Weather conditions are given more importance as it affects the quality of the fruit in its pigmentation and nutritive value. The fruit becomes more vulnerable to diseases that are caused by fungi, bacteria, and viruses due to the climatic changes. Tomato being a berry fruit it has number of attributes such as size, shape, pigmentation, ripening rate to regulate the quality of the fruit. Farmers can benefit from its cultivation only when the fruit has good size, shape, pigmentation, taste and 
ripening rate. Hence it becomes necessary to provide farmers with better aids to detect the diseases that affect the fruit at the early stage [2]. This can prevent the farmer from loss. It can be achieved by implementing image processing techniques in disease detection.

\section{Existing Disease Detection Methods:}

Agriculture being an oldest sector worldwide it is carried out by farmers with the acquired knowledge from their ancestors and experience. Hence, the most common method used in detecting the disease is done by examining the plant with naked eyes. Existing process is time consuming and it may lead to heavy loss for the farmer. To overcome this issue technology can be implemented in the detection and prevention of disease in fruit.

\section{Digital image processing}

Digital image processing is a method that was developed in the 1970s in order to process the simple images. Initially it didn't gain attention as it was not application specific. Later, this methodology gained attention worldwide from the researchers and emerged as system to analyse the images [3][4]. Its applications are not limited to medical imaging, satellite images, videos, agriculture etc., Digital image processing aims to improve the quality of images in order to gain the fine details from the image and to make better solutions for various applications.

\subsection{Image Processing Applied in Agriculture}

At present image processing techniques are applied in agriculture in order to sort

Copyright (C) Author and to grade the vegetables and fruits. The algorithms that are used in this process are used to scan the images and grade the fruit or vegetable based on the attributes such as shape, size and colour. The accuracy of the grading process are affected by the noises that are commonly found in the image. The noises are the outliers that occur during the image acquisition process. The outliers in the image can be removed in the image using filtering techniques [6].

\subsection{Existing Disease Detection Techniques}

Arakeri, M. P. (2016). The author has proposed a method which grades the tomato fruit automatically using computer vision techniques. This automatic grading system is effective in grading the fruit based on the quality of the fruit by analysing the image of the tomato. Noise in the images is removed using preprocessing method The proposed system was able to identify the detective and nondefective fruit with $100 \%$ accuracy and with $96.47 \%$ in detecting ripe/unripe tomatoes. However the prose method has to be enhanced in aspects of speed and accuracy to be implemented in field [13].

Malik, M. H et al(2018). The research work has proposed novel methods in detecting and separating the red mature tomatoes from the background. It is carried out using HSV colour space. The image background is successfully detached with noises and the fruit is detected in the image. Noise in the images is removed using pre-processing method. It is carried out using the watershed method. The proposed method shows good accuracy rate [5]. 
Ferentinos, K. P. (2018).Author has proposed convolutional neural network models in order to detect the fruit disease and diagnose the disease type. The proposed method is trained using the database of 87000 images. The database includes both healthy and unhealthy plant details. The noises in the images were removed using the pre-processing techniques. The performance of the proposed model shows better accuracy rate with $99.53 \%$ [4].

\section{Problem Statement}

The current research focuses in developing a computer aided system in finding the detective region in tomato. It detects the disease found in tomato using image processing techniques. Tomatoes are classified based on the grade i.e infected and uninfected. The proposed application also provides the farmers with necessary information to handle the disease spread at the earliest. It also helps to prevent farmers from heavy loss in production.

\section{Objective}

The current research aims in achieving the following objectives by implementing the image processing and data mining techniques such as:

1. To read the digital image of the tomato fruit and to prepare the image for processing by de-nosing the image using advanced de-noising techniques.

2. To segment the de-noised image tomato fruit image in order to gather the fine details of the image.

3. To extract the required features to segmented image in order to gain the feature vector to support the further analysis.

4. To classify or cluster the tomato fruit based of the category and identify the disease that is found in the fruit.

5. To provide pesticide suggestionbased on the type and severity of the disease.

\section{Proposed Methodology}

The proposed research is carried in six phases as in Figure 1.

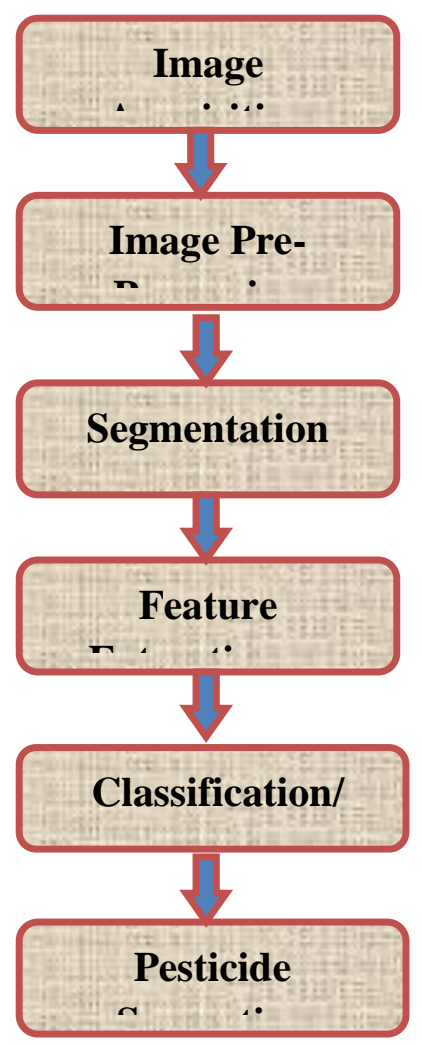

Figure: 1 General Research Flow

\section{Dataset:}

Images of the tomatoes are collected using the Digital cameras with resolution of 16 mega pixels (4920 x 3264pixel resolution) and mobile phones with 13 mega pixels. Images of different disease infected fruits are collected from the local fields in surroundings. 


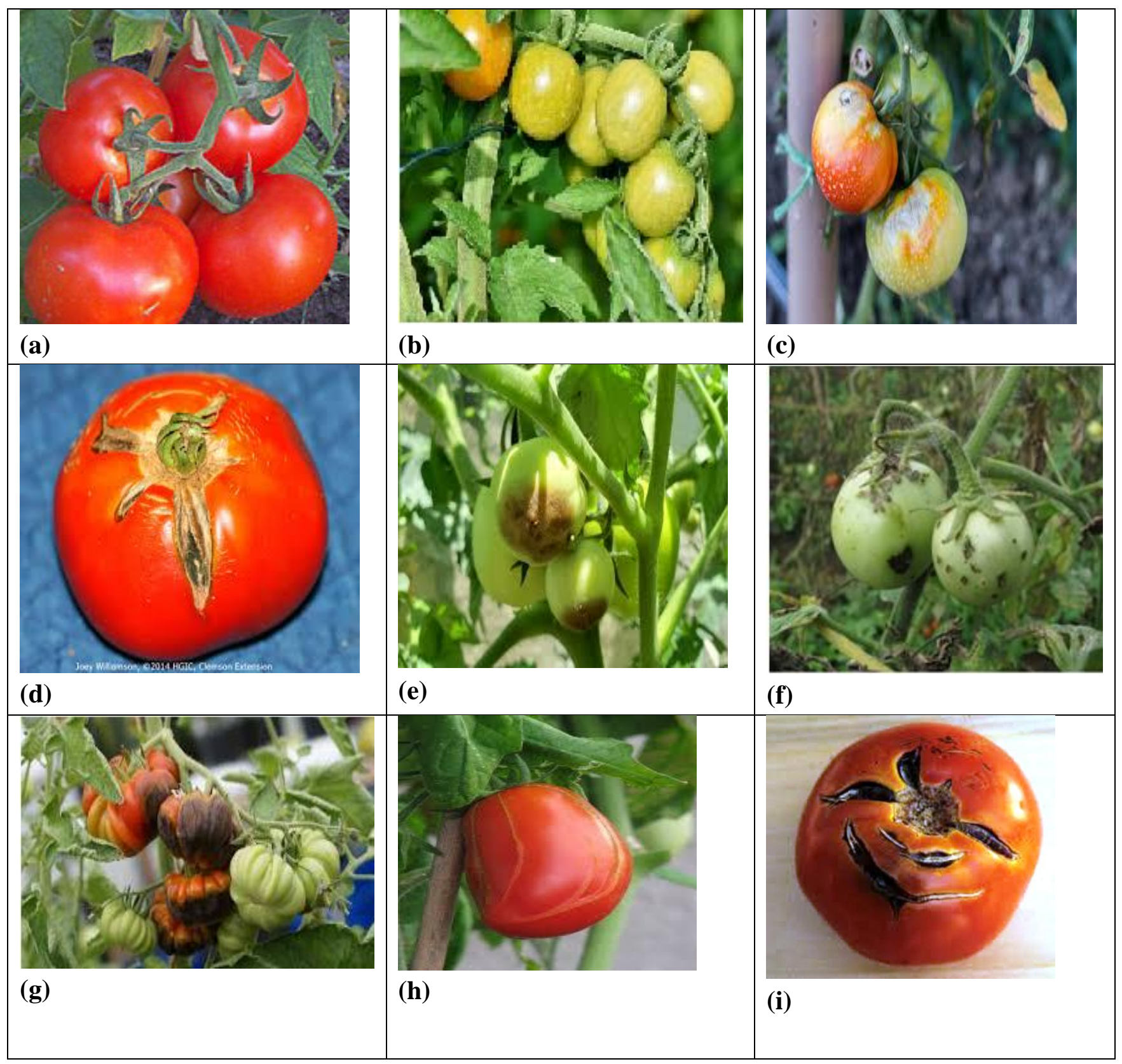

Figure: 2 Data Collection Includes (a)Normal Tomato Fruit,(b) Bacterial Wilt,

(c) Verticillium Wilt, (d)Fruit Cracking,

(e) Early Bottom Rot,(f)Bacterial Spot,

(g) Tomato Blight,(h) Shrink and (i) Puffiness.

Proposed Method(Spiral Seed Filter)

Step 1: Image Acquisition

Step 2: Resize the Image
Step 3: Color conversion: $\mathrm{RGB}$ to $\mathrm{yCbCr}$ and find the luma variance

$\operatorname{Var}\left(\mathrm{Y}^{\prime}\right)=\operatorname{Var}\left(\mathrm{C}_{\mathrm{r}} \cdot\right.$ red $)+\operatorname{Var}\left(\mathrm{C}_{\mathrm{g}}\right.$.green $)+$ $\operatorname{Var}\left(\mathrm{C}_{\mathrm{b}} \cdot\right.$ blue $)+2 \cdot \operatorname{Cov}\left(\mathrm{C}_{\mathrm{r}} \cdot\right.$ red, $\mathrm{C}_{\mathrm{g}} \cdot$ green $)+$ $2 \cdot \operatorname{Cov}\left(\mathrm{C}_{\mathrm{r}} \cdot\right.$ red, $\mathrm{C}_{\mathrm{b}} \cdot$ blue $)+2 \cdot \operatorname{Cov}\left(\mathrm{C}_{\mathrm{g}}\right.$. green, $\mathrm{C}_{\mathrm{b}} \cdot$ blue)

$\operatorname{Var}\left(\mathrm{Y}^{\prime}\right)=\mathrm{C}_{\mathrm{r}}{ }^{2} \cdot \operatorname{Var}($ red $)+\mathrm{C}_{\mathrm{g}}{ }^{2} \cdot \operatorname{Var}($ green $)+$ $\mathrm{C}_{\mathrm{b}}{ }^{2} \cdot \operatorname{Var}($ blue $)+2 \cdot \mathrm{C}_{\mathrm{r}} \cdot \mathrm{C}_{\mathrm{g}} \cdot \operatorname{Cov}($ red, green $)+$ $2 \cdot \mathrm{C}_{\mathrm{r}} \cdot \mathrm{C}_{\mathrm{b}} \cdot \operatorname{Cov}(\mathrm{red}, \quad$ blue $)+$ $2 \cdot \mathrm{C}_{\mathrm{g}} \cdot \mathrm{C}_{\mathrm{b}} \cdot \operatorname{Cov}($ green, blue $)$ 
Step4: $3 \times 3$ horizontal cross-correlation is applied and find the mean and standard deviation of products at each location.

$$
\begin{aligned}
& \text { myvar=zeros([H,1]); } \\
& \text { mystd=zeros([H,1]); } \\
& \text { for } \mathrm{i}=1: \mathrm{H} \\
& \quad \operatorname{diff}=\mathrm{A}(:, \mathrm{i})-\operatorname{colmean}(\mathrm{i}) ; \\
& \operatorname{myvar}(\mathrm{i})=\operatorname{sum}\left(\operatorname{diff} .^{\wedge} 2\right) /(\mathrm{r}-1) \text {; } \\
& \operatorname{mystd}(\mathrm{i})=\operatorname{sqrt}(\operatorname{myvar}(\mathrm{i})) ;
\end{aligned}
$$

end

Step 5: $3 \times 3$ vertical cross-correlation is appliedand find the mean and standard deviation of products at each location.

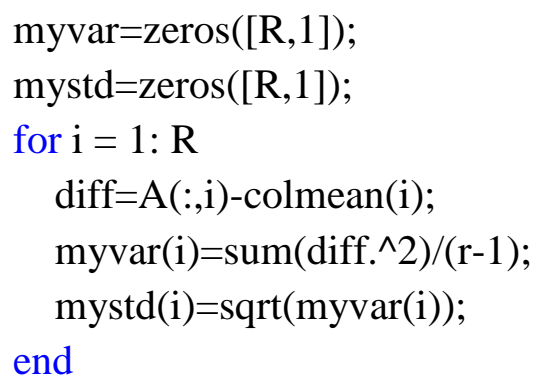

Step6: $4 \times 4$ denoised image is created.

The 10 mega pixels with dimensions $3120 \times 4160$ tomato fruit image is in RGB colour mode captured by camera. Then it is given as input. Then it is resized into 256 x 256 dimensions. Then resized image is converted into $\mathrm{yCbCr}$ colour space to extract the gray image. Then $3 \times 3$ horizontal cross correlation applied. Then $3 \times 3$ vertical cross correlation is applied. Then it produced the $4 \times 4$ denoised output image by computing mean and standard deviation of the $3 \times 3$ nei ghborhood of all pixels in the image.

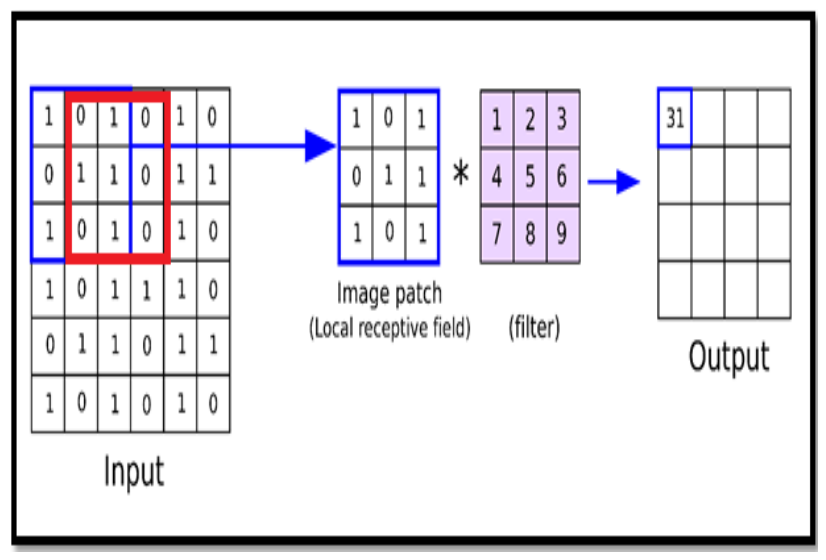

\section{V.RESULT AND DISCUSSION}

The performance of the proposed filters was tested with the five tomato images. The Mean filter and proposed (SSF) filter works well on tomato images corrupted by Salt and pepper noise. From the study on literature, Median filter and Gaussian filters are suitable to remove speckle noise and additive noises. But it is not suitable to remove salt and pepper noise. The results show that the proposed filter removes salt and pepper noise efficiently than other filter. The accuracy of the filter is measured by MSE (Mean Square Error) and PSNR (Peak Signal to Noise)and compared with existing filter. Here, the experimental results are presented for five images in figure3,figure 4 and figure5. The figure 3 shows the input original input image.

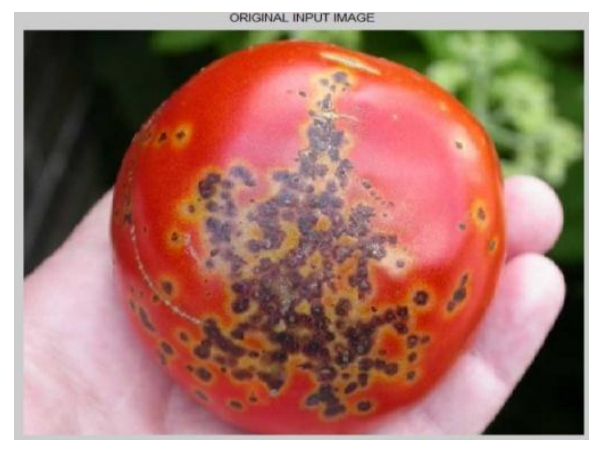

Figure: 3 Input Tomato Image 
The figure 4 shows the color conversion by YCBCR model of input image. In this model Y-luma part is gray scale image. This is separated from CB part and CR part. Then this gray image is used for further analysis.

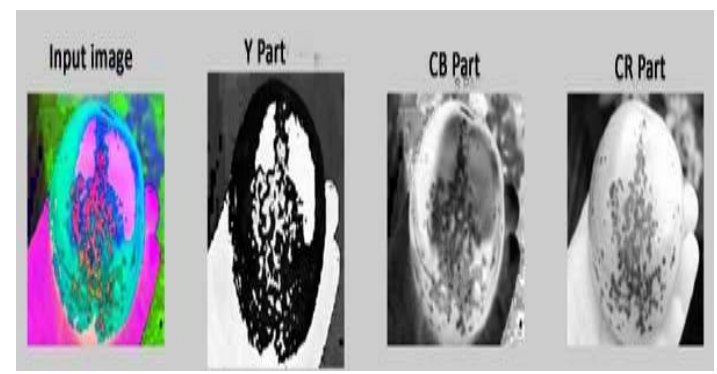

Figure 4. Color conversion using YCBCR The figure 5 shows the result of denoising of input image. The proposed filter gives better result than other filters.

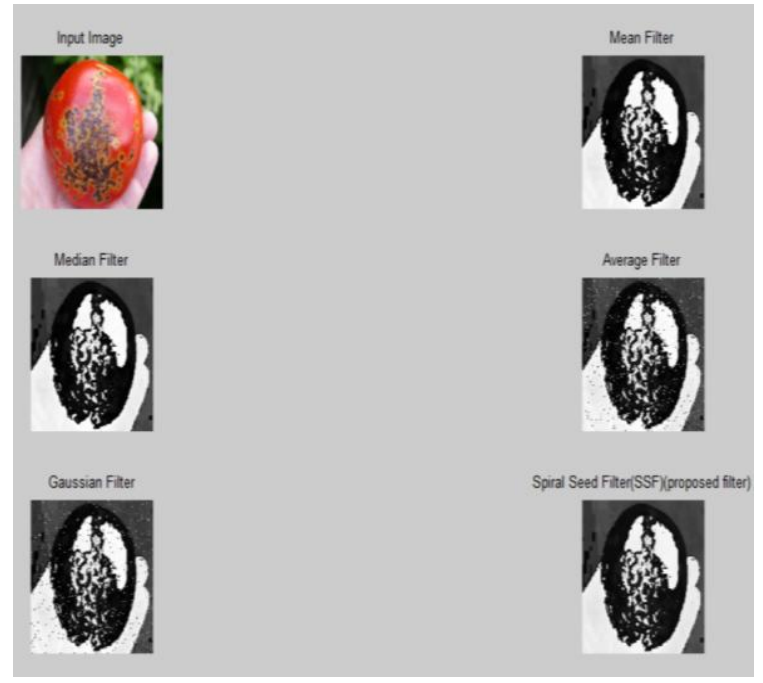

Figure 5. Image Denoising using various filters

Table 1 shows the comparison of PSNR values of Mean, median, gaussain with proposed method (SSF). Highest PSNR value for the Tomato1.jpg of proposed filter is 94.27. The PSNR Value is calculated by using the equation (1).

$$
\begin{aligned}
P S N R & =20 \log _{10} \frac{255}{R M S E} \\
& \rightarrow(1)
\end{aligned}
$$

Copyright (C) Author
RMSE (Root Mean Square Error) is calculated through the square root of MSE.

$$
M S E=\frac{1}{M * N} \sum_{J, K}(f(j, k)-g(j, k))^{2}
$$

$R M S E=\sqrt{M S E}$

(2)

\section{TABLE 1: Comparison of PSNR values}

\begin{tabular}{|l|c|c|c|c|}
\hline Image & $\begin{array}{c}\text { Mean } \\
\text { Filter }\end{array}$ & $\begin{array}{l}\text { Median } \\
\text { Filter }\end{array}$ & $\begin{array}{l}\text { Gaussian } \\
\text { Filter }\end{array}$ & $\begin{array}{c}\text { SSF } \\
\text { (proposed) }\end{array}$ \\
\hline Tomato1.jpg & 85.64 & 74.12 & 64.72 & $\mathbf{9 4 . 2 7}$ \\
\hline Tomat02.jpg & 83.07 & 73.71 & 67.79 & $\mathbf{9 3 . 1 2}$ \\
\hline Tomato3.jpg & 84.21 & 73.53 & 68.41 & $\mathbf{9 2 . 5 7}$ \\
\hline Tomat04.jpg & 80.53 & 74.18 & 65.39 & $\mathbf{9 3 . 6 8}$ \\
\hline Tomat05.jpg & 83.09 & 73.43 & 64.02 & $\mathbf{9 3 . 5 4}$ \\
\hline
\end{tabular}

Table 2 shows the comparison of MSE values of mean, median, gaussian with proposed method (SSF). Proposed filter acquired minimum error value than other existing method.

TABLE2: Comparison of MSE values

\begin{tabular}{|l|l|l|l|l|}
\hline Image & $\begin{array}{l}\text { Mean } \\
\text { Filter }\end{array}$ & $\begin{array}{l}\text { Median } \\
\text { Filter }\end{array}$ & $\begin{array}{l}\text { Gaussian } \\
\text { Filter }\end{array}$ & $\begin{array}{l}\text { SSF } \\
\text { Filter }\end{array}$ \\
\hline Tomato1.jpg & 1.8601 & 1.0025 & 1.2208 & $\mathbf{0 . 0 0 0 1}$ \\
\hline Tomat02.jpg & 1.9034 & 1.0028 & 1.1090 & $\mathbf{0 . 0 0 0 1}$ \\
\hline Tomat03.jpg & 1.8001 & 1.0029 & 1.0945 & $\mathbf{0 . 0 0 0 1}$ \\
\hline Tomat04.jpg & 1.7931 & 1.0025 & 1.1193 & $\mathbf{0 . 0 0 0 0}$ \\
\hline Tomat05.jpg & 1.8103 & 1.0030 & 1.2595 & $\mathbf{0 . 0 0 0 2}$ \\
\hline
\end{tabular}

\section{CONCLUSION}

This research proposed a new Spiral Seed Filter(SSF) and compared with other three existing filters. In this research various types of filters have described and studied the filters used in various investigators [11]. The proposed filter conserves the edges and decreases the computation time. Experimental result shows high PSNR 
such as $94 \%$. It specifies proposed filter improved the quality of tomato images than the other filters such as Mean, Median and Gaussian filters. It gives low value of $\operatorname{MSE}(0.0001)$ than others. It indicates the better enhancement of images than other methods.

\section{References}

1.Rajni, Pawan Kumar Dahiya (2020), “A comparative analysis of image enhancement techniques", Journal of Xi'an University of Architecture \& Technology. 2.Linwei Fan et al. (2019),"Brief review of image denoising techniques", Visual Computing for Industry, Biomedicine and Art .

3. Diwakar M, Kumar M (2018), "A review on $\mathrm{CT}$ image noise and its denoising", Biomed Signal Process Control 42:73-88.

4. Ferentinos, K. P. (2018), "Deep learning models for plant disease detection and diagnosis" Computers and Electronics in Agriculture, 145, 311-318.

5.Malik, M. H., Zhang, T., Li, H., Zhang, M., Shabbir, S., \& Saeed, A. (2018), "Mature tomato fruit detection algorithm based on improved HSV and watershed algorithm”, IFAC-PapersOnLine, 51(17), 431-436.

6. M. M. Puranik1 S.V.Halse (March 2018), "A Review Paper: Study of Various Types of Noises in Digital Images", International Journal of Engineering Trends and Technology (IJETT).

7. Oo, Y. M., \& Htun, N. C. (2018), "Plant leaf disease detection and classification using image processing", International Journal of Research and Engineering, 5(9), 516-523.
8. Zhe Liu. et al. (2018), "Image Denoising Based on A CNN Model", International Conference on Control, Automation and Robotics.

9. Ms. Shefali A. et al. (2017), "Review Paper on Image Denoising Techniques", International Research Journal of Engineering and Technology.

10. Sheeraz Ahmed Solangi. et al. (2017), "Image Denoising Methods: Literature Review", International Journal of Recent Research and Applied Studies.

11. Astrit Hulaj. Et al (2017), "Removal of various noises from Digital images with the application of a single algorithm", International Journal of Civil Engineering and Technology.

12. Raut, S., \& Ingole, K. (2017), "Review on leaf disease detection using image processing techniques", International Research Journal of Engineering and Technology, 4(04), 2044-2047.

13. Arakeri, M. P. (2016), "Computer vision based fruit grading system for quality evaluation of tomato in agriculture industry “, Procedia Computer Science, 79, 426-433.

14.Mokhtar, U., El Bendary, N., Hassenian, A. E., Emary, E., Mahmoud, M. A., Hefny, H., \& Tolba, M. F. (2015), "SVM-based detection of tomato leaves diseases" , In Intelligent Systems' 2014 (pp. 641-652). Springer, Cham.

15.Mokhtar, U., Ali, M. A., Hassenian, A. E., \& Hefny, H. (2015), "Tomato leaves diseases detection approach based on support vector machines" In 2015 11th International Computer Engineering Conference (pp. 246-250). IEEE. 